\title{
Effect of wave propagation and transfer of a sensor on detected acoustic emission signal
}

\author{
Marek Raček ${ }^{1, *}$ \\ ${ }^{1}$ Faculty of Mechanical Engineering STU Bratislava, Institute of applied mechanics and mechatronics, \\ Nám. Slobody 17 Bratislava, Slovakia
}

\begin{abstract}
One of the biggest challenge for acoustic emission (AE) signal classification on real constructions is transfer from laboratory measurement on specimens to full scale. Detected AE responses are (among other factors) strongly influenced by propagation of waves in material and transfer function of sensor used. Propagation of elastic stress waves in plate of AISI 304 stainless steel was investigated in this work. Two types of wave excitation were used: broad band HSU-Nielsen source and artificial narrow band source via Vallen VS150-M transducer. AE responses were detected with use of 3 different $\mathrm{AE}$ transducers to take into account the effect of sensor transfer function on detected response. Attenuation curves were constructed from extracted amplitudes and digitalized signal was recorded for subsequent advanced analysis. Description of detected wave modes and separation of reflected waves was successfully done with use of combination of voltage-time dependency, wavelet transform (WT) and dispersion curves. Effect of wave propagation and transfer function of a sensor on signal maximum amplitude was discussed.
\end{abstract}

Keywords: Acoustic emission, Lamb waves, Dispersion curves, Wavelet analysis

\section{Introduction}

$\mathrm{AE}$ is the phenomenon whereby transient elastic waves are generated by the rapid release of energy from localized sources within a material [1]. AE method should be considered to be a passive non-destructive technique [2]. Employment of AE testing is diverse ranging from traditional industrial detection of defects in vessel and pipes to special applications with capability of source type classification, pharmaceutical applications, biologic and bionic applications, etc. Especially the distinction of different source types is of huge interest in the field of industrial integrity assessment, anyway this capability of AE is still reduced only on some special applications.

Challenges of accurate location and characterisation of AE signals are related to transfer path between source mechanism and digitised signal. The form of captured AE signal depends upon the wave propagation path from the source to the sensor, the coupling of the

${ }^{*}$ Corresponding author:

Reviewers: Marcin Kubiak, Ján Vavro 
sensor, the transfer function of individual sensors and the acquisition hardware. Changing any of these dependencies will result in a different form of recorded signal from an identical source mechanism [3]. The wave propagation in various structures can be multimodal, dispersive and subject to attenuation. Sensors usually employed to capture AE waves are contact lead zirconate titanate (PZT) transducers and can be resonant or broad-band. The aim of this paper is to analyse the influence of wave propagation and transfer of a sensor on the captured AE signal. Set of experiments was conducted on $15 \mathrm{~mm}$ thick plate of AISI 304 stainless steel (SS). Results will serve as a reference for the following research considering signal classification in pressure vessel from same material type and wall thickness.

\section{Theory of guided waves}

\subsection{Guided waves in plates}

Guided waves, unlike of bulk waves, are characterized by continuous interaction with boundary of material which can create a new wave structure. As an incident wave encounters a boundary both reflected wave of a same mode and reflected wave of converted mode can be emanated back into the material. The wave structure in plates consists of multiple reflections between free surfaces of plate [4]. These waves which can exist in isotropic elastic plates of finite thickness are the Lamb waves [5].

The governing equations for both guided and bulk waves are the same and can be derived from equation of motion with absence of body force by utilizing Hooke's law. Assuming an isotropic material, the governing equation can be expressed in terms of displacements as follows [6]:

$$
\mu \nabla^{2} \mathbf{u}+(\lambda+\mu) \boldsymbol{\nabla}(\boldsymbol{\nabla} \cdot \mathbf{u})=\rho \ddot{\mathbf{u}}
$$

where $\mathbf{u}$ is the displacement vector, $\ddot{\mathbf{u}}$ its second derivative with respect to time, $\lambda$ and $\mu$ are Lamé constants, $\boldsymbol{\nabla}$ is the nabla differential operator, and $\rho$ is the mass density. In case of plate waves, traction free boundary conditions need to be satisfied on free surfaces of a plate. Considering two-dimensional plate depicted on Fig. 1 with coordination system positioned on midplane of plate, boundary conditions for Lamb waves takes the form:

$$
\sigma_{z}=\tau_{z x}=0 \text { for } z= \pm h .
$$

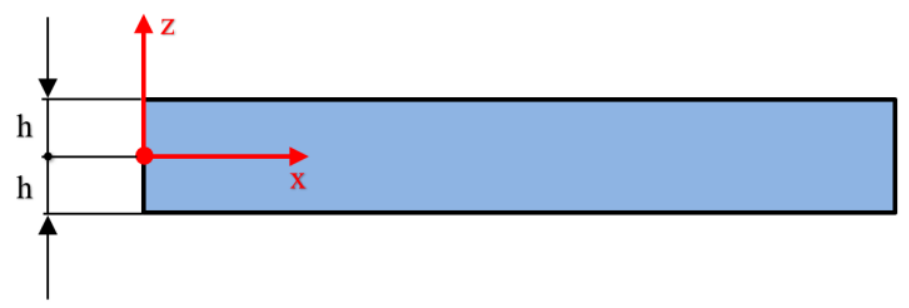

Fig. 1. Geometry of two-dimensional plate

\subsection{Dispersion curves}

Known phenomenon related to the wave propagation is dispersion. In general case, dispersion refers to frequency dependence of wave velocity and is mainly caused by non-linear elastic behaviour of the material [7]. Dispersion curves as a functional dependencies of wave phase 
velocities on frequency can be derived from eq. (1) using Helmholtz decomposition of displacement vector $[4,6]$ :

$$
\mathbf{u}=\boldsymbol{\nabla} \phi+\nabla \times \Psi
$$

where $\phi$ is a scalar function and $\boldsymbol{\psi}$ is the zero divergence vector. By use of this approach, called the method of displacement potentials, the governing equations for longitudinal and shear waves can be yielded:

$$
\begin{aligned}
& c_{L}^{2} \nabla^{2} \phi=\ddot{\phi} \\
& c_{T}^{2} \nabla^{2} \boldsymbol{\psi}=\ddot{\boldsymbol{\psi}}
\end{aligned}
$$

where $c_{L}$ and $c_{T}$ stands for longitudinal and transverse wave velocity. Assuming infinite plane harmonic wave solutions of equations (4), (5) and applying boundary conditions, RayleighLamb frequency equations for symmetric and antisymmetric Lamb wave modes can be obtained:

$$
\begin{aligned}
& \frac{\tan (q h)}{\tan (p h)}=-\frac{4 k^{2} p q}{\left(q^{2}-k^{2}\right)^{2}} \\
& \frac{\tan (q h)}{\tan (p h)}=-\frac{\left(q^{2}-k^{2}\right)^{2}}{4 k^{2} p q}
\end{aligned}
$$

where:

$$
\begin{aligned}
& p^{2}=\left(\frac{\omega}{c_{L}}\right)^{2}-k^{2} \\
& q^{2}=\left(\frac{\omega}{c_{T}}\right)^{2}-k^{2}
\end{aligned}
$$

$\omega$ is the angular frequency and $\mathrm{k}$ is the wavenumber. For a given $\omega$ the finite number of real $k$ can be computed solving eq. (6), (7). Finally, phase velocity dispersion curves can be derived from relation between phase velocity, wavenumber and angular frequency:

$$
c_{p}=\frac{\omega}{k}
$$

\subsection{Group velocity}

Other remarked phenomenon related to the wave propagation is that waves advance in groups. By observing of waves propagating in plates the groups of waves of similar frequencies travel with so called group velocity rather than phase velocities of its single components. The group velocity can be expressed by [8]: 


$$
c_{g}=\frac{d\left(k c_{p}\right)}{d k}
$$

thus group velocity dispersion curves can be derived from phase velocity dispersion curves.

\section{Experimental procedure}

\subsection{Experiment set-up}

The experimental measurements were carried on plasma cut section of $15 \mathrm{~mm}$ thick plate of AISI $304 \mathrm{SS}$ with final lateral dimensions $0.75 \mathrm{~m}$ by $1.35 \mathrm{~m}$. Surface displacements were captured using standard contact piezo transducers coupled by Vallen acoustic grease. Three types of sensors were used: broadband DWC B225.5 $(50-400 \mathrm{kHz})$ manufactured by Digital Wave Corporation, broadband VS45-H $(20-450 \mathrm{kHz})$ and low frequency VS30-V $(25-80$ $\mathrm{kHz}$ ) by Vallen Systeme GmbH. Signal from sensors was preamplified by Vallen AEP5 preamplifier with $35 \mathrm{~dB}$ gain and carried to Vallen AMSY-6 AE system. Ultrasonic waves were excited by broadband Hsu-Nielsen $(\mathrm{H}-\mathrm{N})$ source, which is standardized source for evaluation of wave propagation effect [9]. In case of wideband sensors (B225.5, VS45H), waves were also excited by artificial narrow band source in order to evaluate the effect of wave propagation in narrow frequency band. Artificial source was represented by Vallen VS150-M transducer driven by 5 cycle sinusoidal burst with central frequency of $150 \mathrm{kHz}$, which has been used in many works investigating the propagation of guided waves [10-12].

Set of experiments was conducted for each source-sensor distance ranging from $20 \mathrm{~mm}$ to $600 \mathrm{~mm}$. Position of the sensor was held constant and the source position varied, in order to obtain particular source-sensor distance. Extracted AE parameters were saved for each experiment realization and one waveform was recorded for each source-sensor distance.

\subsection{Data processing}

Although fully digitalized captured signal carries most possible information about measured quantity, simple waveform of the signal sometimes does not provide sufficient information about wave propagation. In contrary, by employing of WT, the time dependency of frequency content of a signal can be obtained, and thus WT provide valuable information mostly about dispersive nature of waves which was proved by several researches [13-16]. In presented paper, continuous wavelet transform (CWT) of each digitized signal was computed by the cwt function in Matlab software with use of analytic Morse wavelet and time-bandwidth product equal to 60 . From CWT scalogram the distribution of signal's energy can be discriminated in both time and frequency domain Fig. 2. 


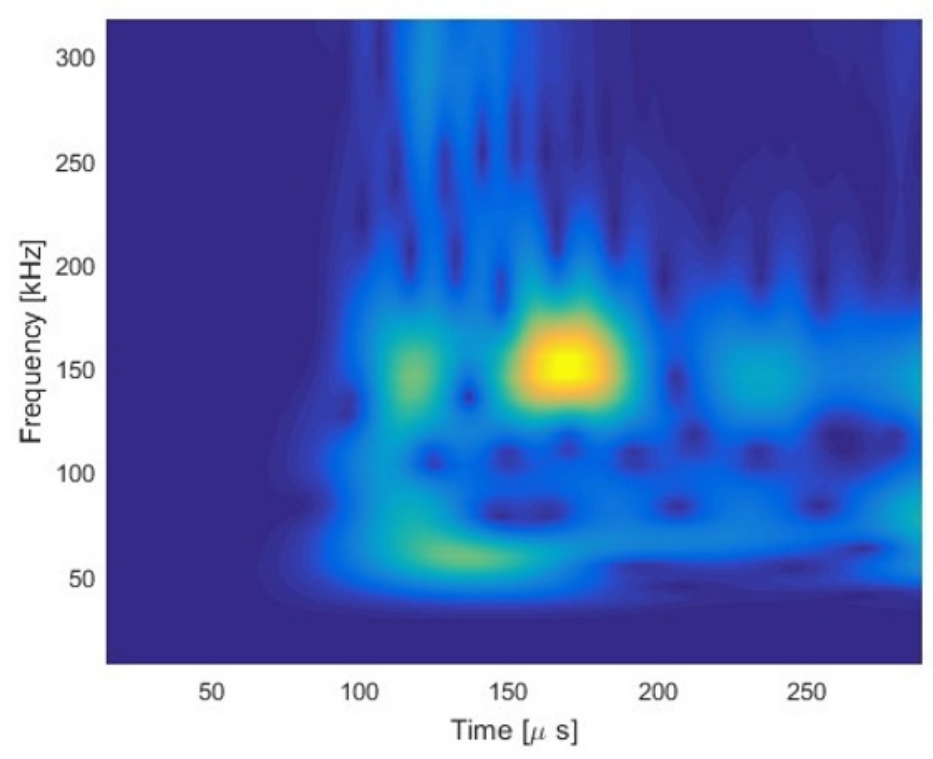

Fig. 2. Computed CWT

Transcendental Rayleigh-Lamb equations (6), (7) has been solved numerically by change of sign method with separation of roots corresponding to discontinuity. Finite number of real wavenumbers was found for each angular frequency. The group velocity dispersion curves (depicted on Fig.3) were computed for usable frequency range of each sensor.

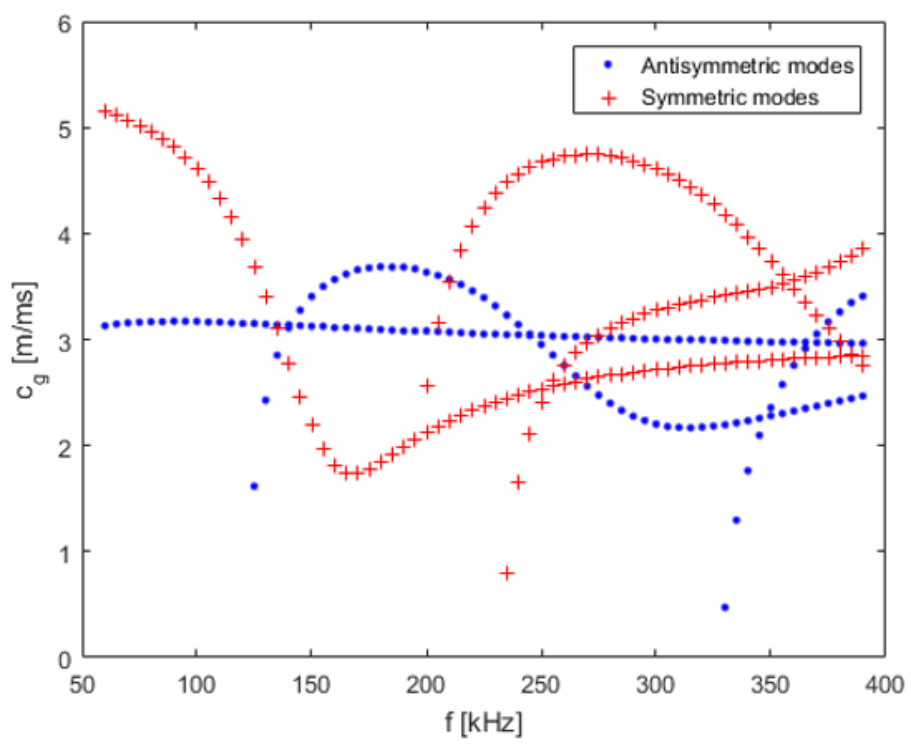

Fig. 3. Group velocity dispersion curves in frequency range of the sensor DWC B225.5

In order to assign time-frequency domains to particular Lamb wave modes, group velocity dispersion curves were recalculated to time-delays relative to reference group and plotted to CWT scalogram. Reference group was as a rule selected A0 of frequency above $60 \mathrm{kHz}$, 
where the group velocity does not differ significantly with frequency. This reference group should be at first manually recognized in scalogram, according to some experience, in order to assign the time of arrival corresponding to CWT computational time. Subsequently from known time of arrival of reference group, source-sensor distance and group velocity dispersion curves, CWT scalogram and dispersion curves were merged. Time-frequency domains corresponding to particular modes were selected manually as the intersections of dispersion curves with distinguishable CWT areas (Fig. 4).

In plate of finite lateral dimensions, interaction of Lamb waves with plate edges needs to be considered. The reflected wave field from the free edge is assumed to be a superposition of all modes of an infinite plate and is governed by Snell's law [17]. Oblique incidence of Lamb waves is a vast research area. In range of this work, focus was reduced to two direct and one oblique incidence assuming reflected mode is the same as incident and the angle of incidence is equal to the angle of reflection. Dispersion curves for reflected waves were plotted to CWT scalogram (Fig. 5) using the methodology described above, but source-sensor distance was substituted by distance that incidence wave plus reflected wave travels.

According to standard [9] amplitude attenuation curves were constructed in linearized form and divided into 2 regions - near field and far field. Attenuation curves as dependency of amplitude decrease on distance were constructed from amplitudes automatically extracted by AE system. The set of amplitudes was obtained for each source-sensor position and final amplitude in the particular position was computed as an average value. One saved waveform of the signal for each source-sensor distance served for deeper analysis of signal.

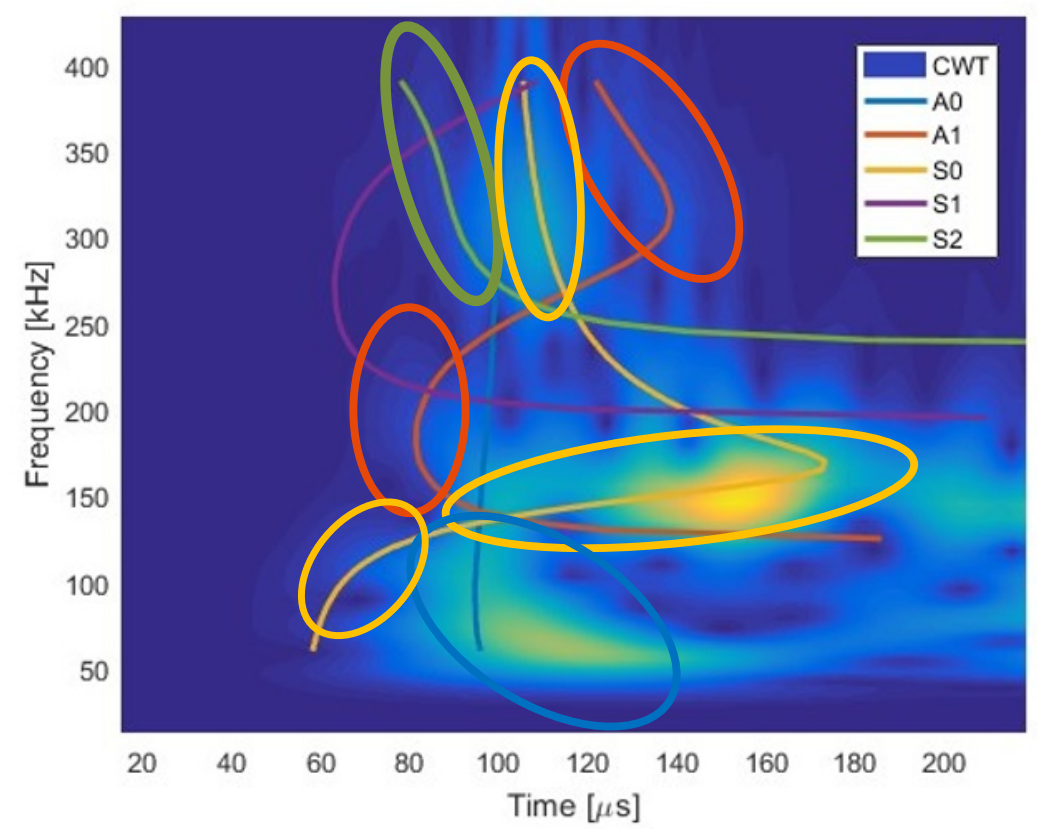

Fig. 4. Group velocity dispersion curves plotted in CWT scalogram. Selected time-frequency domains are marked with ellipses of colour corresponding to mode colour in legend 


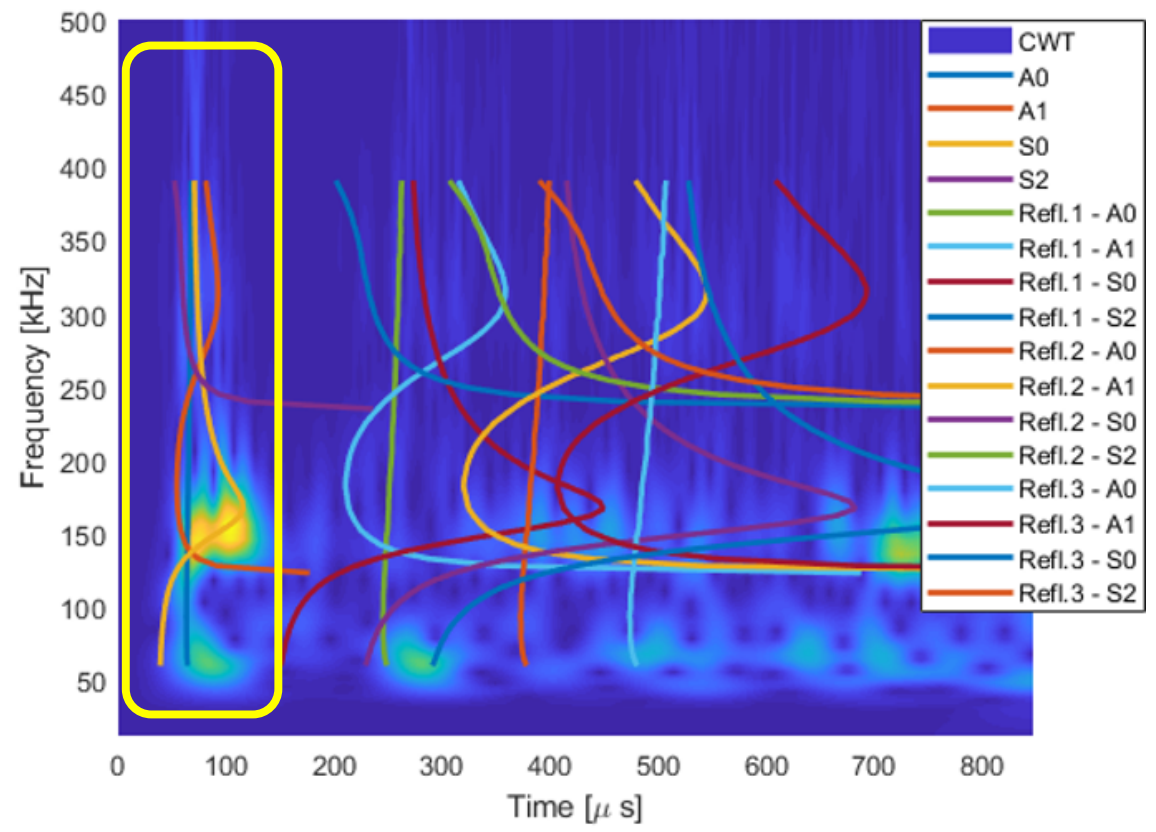

Fig. 5. Dispersion curves for direct and reflected waves plotted in CWT scalogram. Direct wave separated from reflected waves by yellow border

\section{Results and discussion}

Typical CWT and dispersion curves corresponding to measured signals from sensors B225.5, VS30V and VS45H in distance of $250 \mathrm{~mm}$ from H-N source position are depicted on Fig. 6. In order to relate the dominate modes detected by particular sensor type, sensitivity curves for each sensor are plotted on Fig. 7.
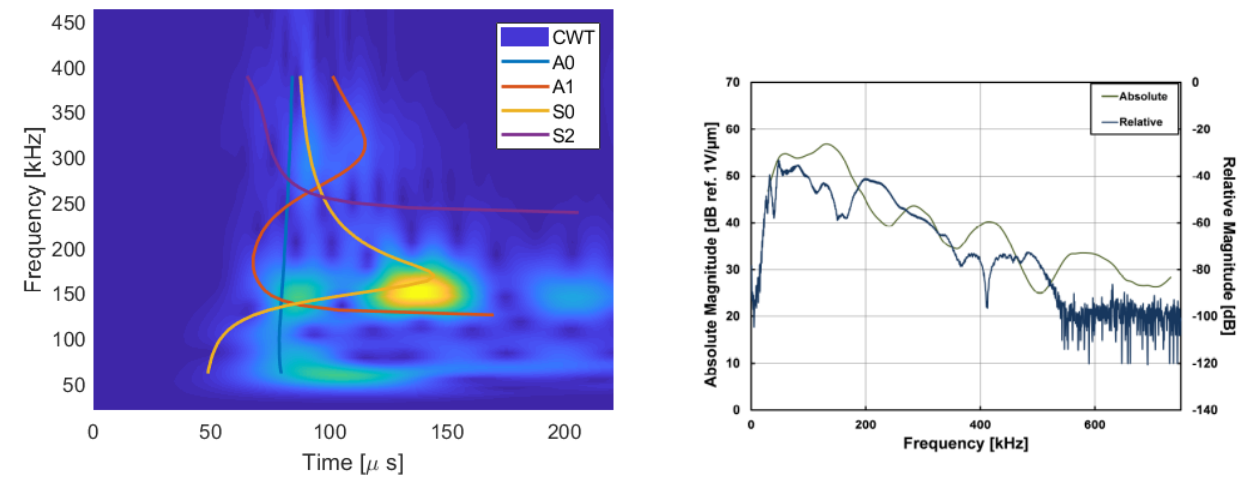

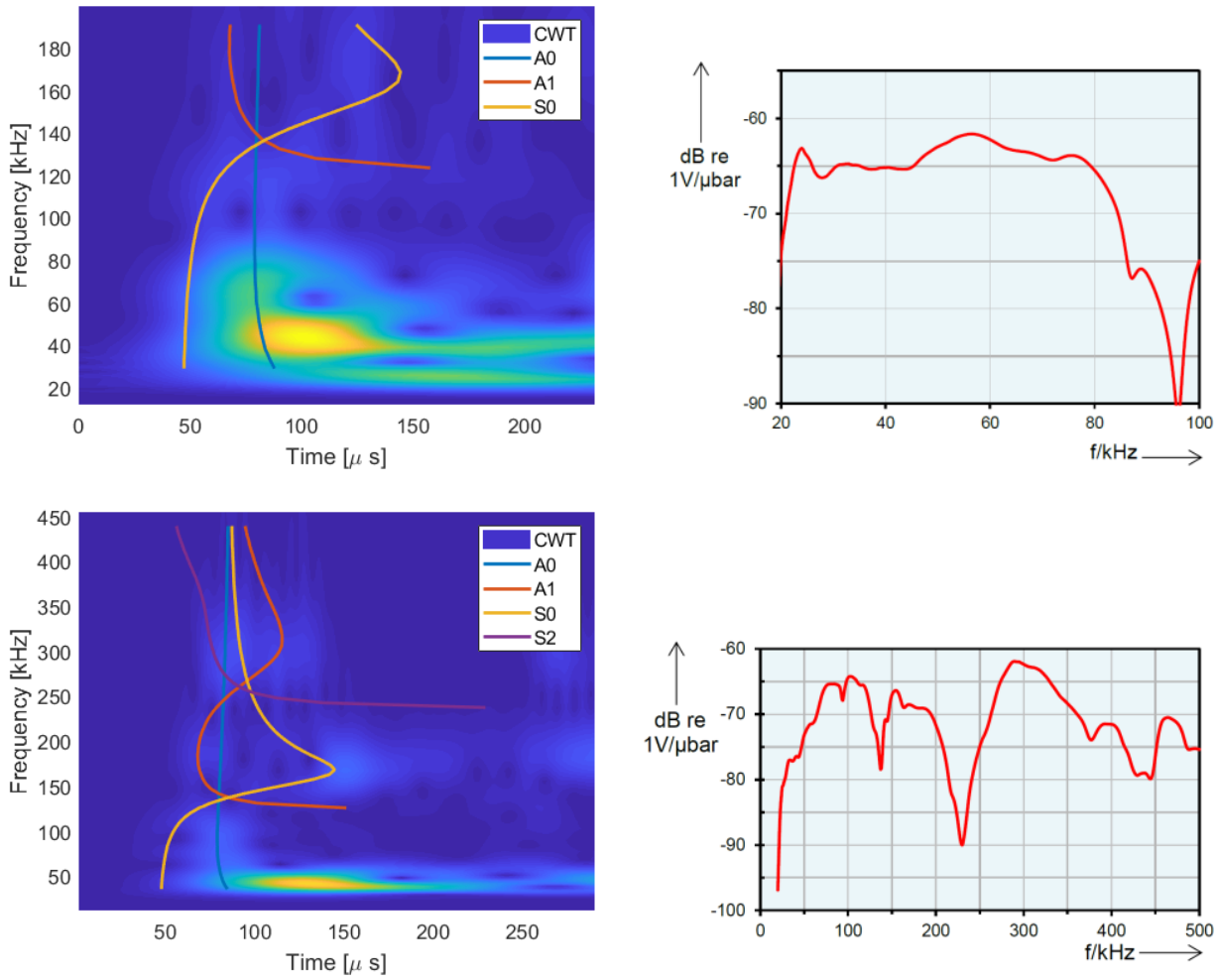

Fig. 6. Typical CWT and dispersion curves. Source: H-N, sensors: B225.5(above), VS30V (middle), VS45H (bottom)

Fig. 7. Sensitivity curves of sensors: B225.5 (above), VS30V (middle), VS45H (bottom)

Detected modes, modes and frequencies corresponding to maximum amplitude and modes which vanish after some propagation distance are listed in Table 1 for sensors B225.5 and VS30V.

Table 1. Detected modes, attenuated modes and modes of maximum amplitude

\begin{tabular}{|c|c|c|c|c|}
\hline Sensor & Source & Detected modes & $\begin{array}{c}\text { Attenuated } \\
\text { modes } \\
\text { (distance) }\end{array}$ & $\begin{array}{c}\text { Mode(frequency) } \\
\text { of maximum }\end{array}$ \\
\hline \multirow{3}{*}{ B225.5 } & $\mathrm{H}-\mathrm{N}$ & $\mathrm{A} 0, \mathrm{~S} 0, \mathrm{~A} 1, \mathrm{~S} 1$, & $\mathrm{S} 1(200 \mathrm{~mm})$ & $\begin{array}{c}\mathrm{S} 0(150 \mathrm{kHz}) / \\
\mathrm{A} 0+\mathrm{S} 0+\mathrm{A} 1 \\
(135 \mathrm{kHz})\end{array}$ \\
\cline { 2 - 5 } & $\mathrm{VS} 150 \mathrm{M}$ & $\mathrm{A} 0, \mathrm{~S} 0, \mathrm{~A} 1$ & - & $\mathrm{S} 0(150 \mathrm{kHz})$ \\
\hline $\mathrm{VS} 30 \mathrm{~V}$ & $\mathrm{H}-\mathrm{N}$ & $\mathrm{A} 0, \mathrm{~S} 0,(\mathrm{~A} 1)$ & - & $\mathrm{A} 0(40 \mathrm{kHz})$ \\
\hline
\end{tabular}

Dominant frequency areas and modes of detected signal can be explained by nature of Lamb waves where particle displacements are composed from in plane $(\mathrm{u})$ and out of plane (w) displacements, and nature of contact PZT transducers which can detect only surface vibrations and is preferable sensitive to out of plane displacements. This means, that dominant modes and frequencies of a signal correspond to those modes and frequency areas of which vibration on surface is preferably in $\mathrm{z}$ direction and are situated in proximity of sensor's sensitivity peak. Normalized through thickness displacements calculated for a plate of $15 \mathrm{~mm}$ thick AISI $304 \mathrm{SS}$ are depicted on Fig. 8 for S0 mode and Fig. 9 for A0 mode. 

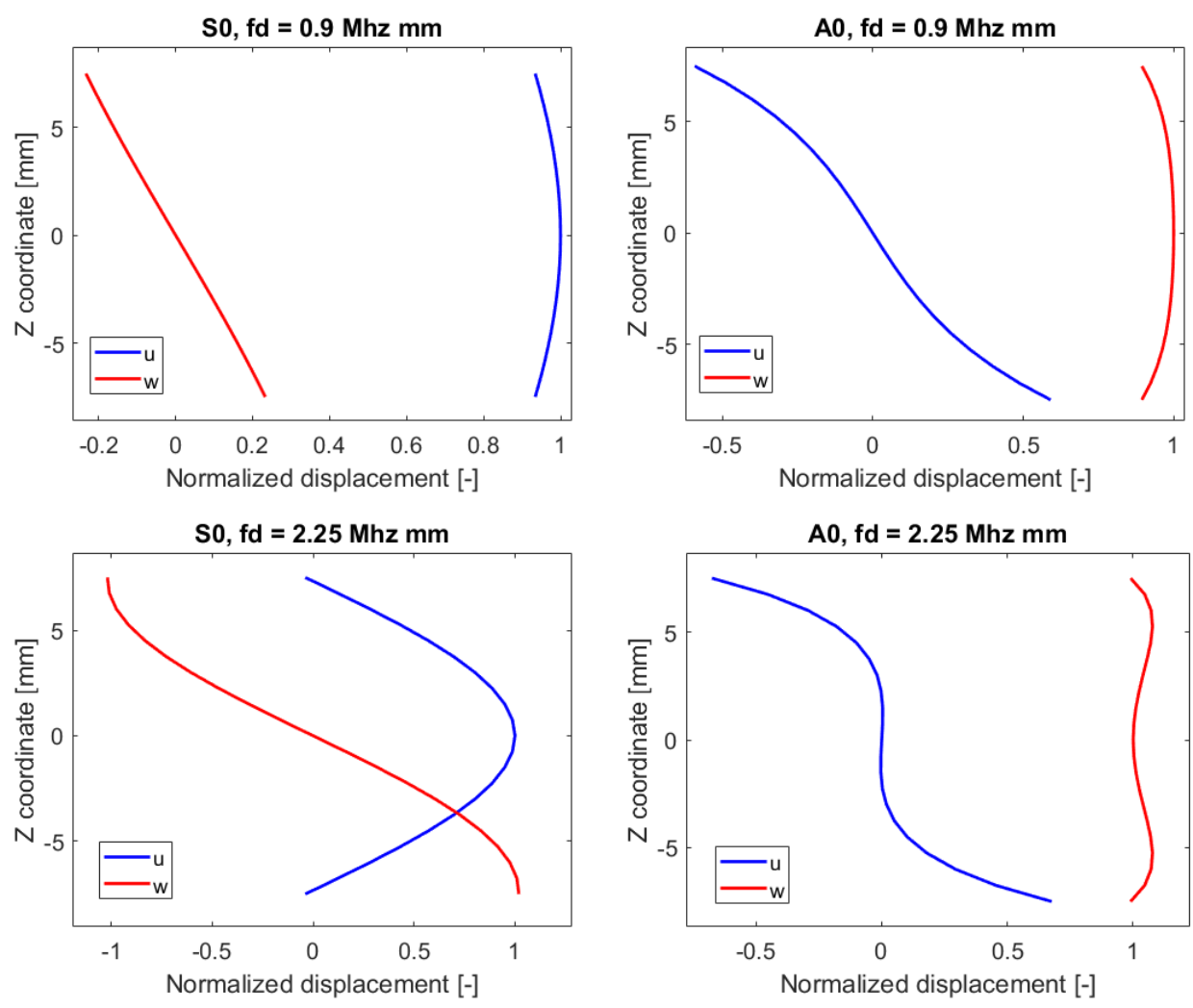

Fig. 8. Through thickness displacement, mode $\mathrm{S} 0$ at frequencies $60 \mathrm{kHz}$ (above), $150 \mathrm{kHz}$ (bottom)

Fig. 9. Through thickness displacement, mode $\mathrm{A} 0$ at frequencies $60 \mathrm{kHz}$ (above), $150 \mathrm{kHz}$ (bottom)

It can be seen, that in the lower frequency band, very weak signal of S0 mode can be captured, because almost whole vibration of S0 takes place in $\mathrm{x}$ direction. The dominant mode in proximity of $60 \mathrm{kHz}$ is $\mathrm{A} 0$ of which displacement in $\mathrm{z}$ direction is greater than in $x$ direction.

Detected dominant mode of sensor VS30V, sensitive to frequencies below $80 \mathrm{kHz}$, is A0 which is in accordance with analysis above. On frequencies around $150 \mathrm{kHz}$, where B225.5 sensor has a sensitivity peak, S0 vibrates almost exclusively in $\mathrm{z}$ direction. Also experimental results reveals, that dominant mode captured by B225.5 sensor appertains to S0 in proximity of $150 \mathrm{kHz}$. Note, that the mode of maximum amplitude in case of excitation by $\mathrm{H}-\mathrm{N}$ source varies: S0 (150 kHz), resp. common arrival of S0 + A0 + A1 $(135 \mathrm{kHz})$. Preferred mode for maximum amplitude depends upon phase shift of modes S0, A0, A1 in proximity of $135 \mathrm{kHz}$. In contrary when narrow band source via VS150M sensor with energy concentrated on frequency of $150 \mathrm{kHz}$ used, maximum amplitude corresponds only to S0 mode. Typical CWT and dispersion curves of this kind of signal is depicted on Fig. 10 for source-sensor position of $250 \mathrm{~mm}$. 


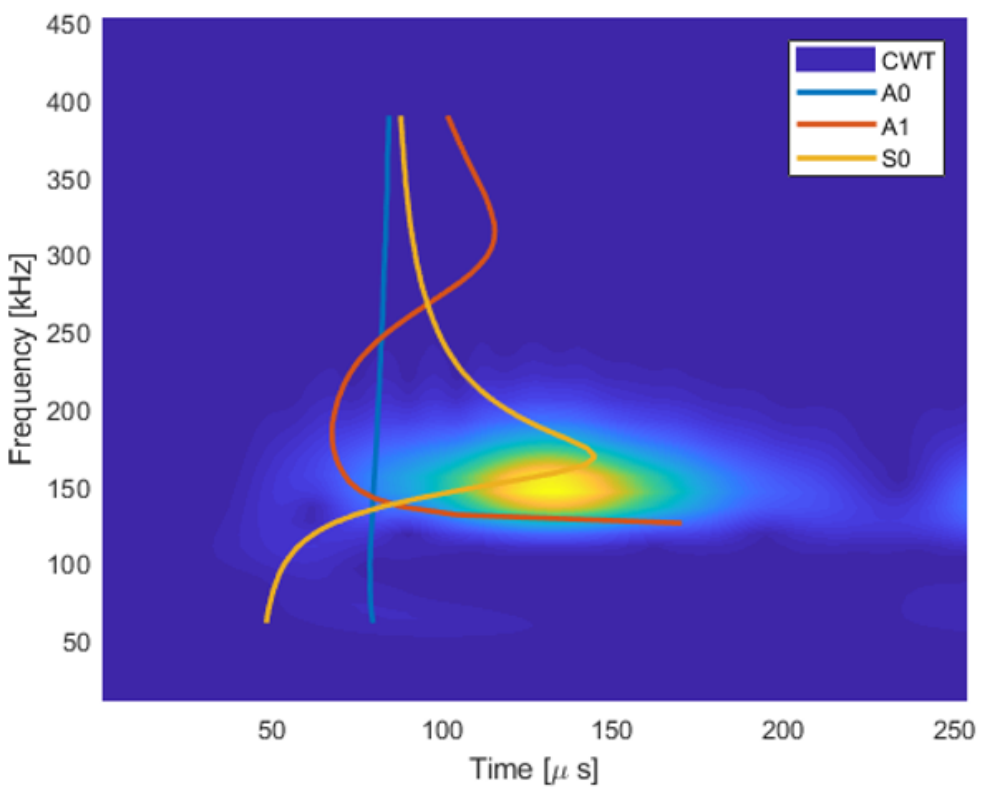

Fig. 10.CWT and dispersion curves of sensor B225.5, source VS150M

In the case of VS45H sensor, with dominant frequencies in proximity of $100 \mathrm{kHz}, 150$ $\mathrm{kHz}$, and $300 \mathrm{kHz}$, detected signal occurred mostly bellow $50 \mathrm{kHz}$ (Fig. 6 bellow) which is a typical case of flawed coupling to plate. Sensor VS45H was excluded from current study, but one interesting phenomenon was revealed. Although mentioned sensor was obviously wrong coupled and very poor signal was detected in frequency band above $50 \mathrm{kHz}$ for each source-sensor position, maximum amplitude of $\mathrm{H}-\mathrm{N}$ source in close proximity of the sensor exceeded $90 \mathrm{~dB}$. Thus if this was a case of AE measurement on real construction, coupling and sensitivity of the sensor would be classified as good, according to standard [9]. This demonstrates the significance and additional value of advanced processing of measured $\mathrm{AE}$ signals.

Linearized attenuation curves constructed from extracted amplitudes are depicted on Fig. 11. According to standard [9] approximated near field / far field border is situated in $20 \times$ thickness, which corresponds to $300 \mathrm{~mm}$. By subsequent advanced analysis of recorded signals it was revealed, that in case of B225.5 sensor, maximum amplitudes in positions above $250 \mathrm{~mm}$ correspond to superposition of reflections. Similarly, amplitudes extracted from VS30V sensor above $300 \mathrm{~mm}$ did not appertain to primary wave. Thus only linearized near field can provide relevant information about wave attenuation in this particular case. Note, that attenuation of signal captured by B225.5 sensor is more pronounced than of signal captured by VS30V sensor. In case of VS30V, maximum amplitude corresponds to A0 mode, which is not subjected to frequency dispersion as significantly as S0 mode. In addition to dispersion attenuation mechanism, absorption and scattering increase with frequency. With respect to all mentioned attenuation mechanisms, VS30V preferable provide less attenuated signal. In order to construct relevant attenuation curve for the far field the maximum amplitudes from waveform after separation of reflections can be used. Unfortunately, a set of recorded waveforms for each source-sensor distance would be required for this purpose because of well-known weak reproducibility of $\mathrm{H}-\mathrm{N}$ source in terms of maximum amplitude. 

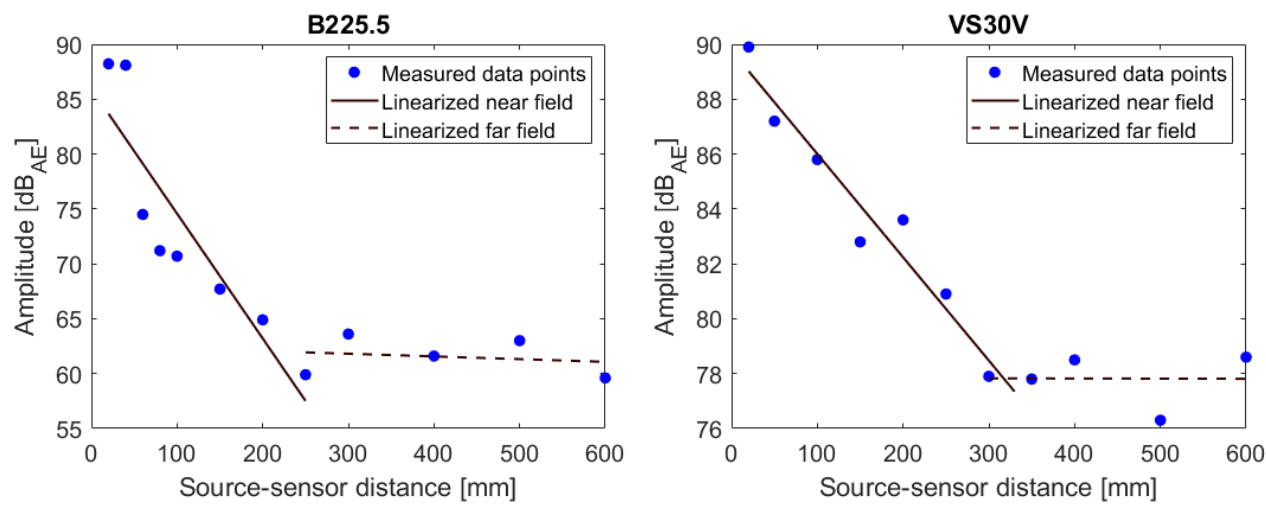

Fig. 11.Attenuation curves

Significant AE parameter related to position of maximum amplitude is rise time. With knowledge of mode and time of maximum amplitude, linear dependency of rise time on source-sensor distance can be obtained for pulse like signals - Fig. 12. In the case of sensor B225.5 and H-N source 2 lines were constructed to appropriate approximate rise times, one to fit time positions of maximums corresponding to S0 mode and another one to fit maximums of $\mathrm{S} 0+\mathrm{A} 0+\mathrm{A} 1$ modes. Slope of the curve can serve for correction of rise time with distance. Experimentally and theoretically stated slopes of rise time vs. distance curve
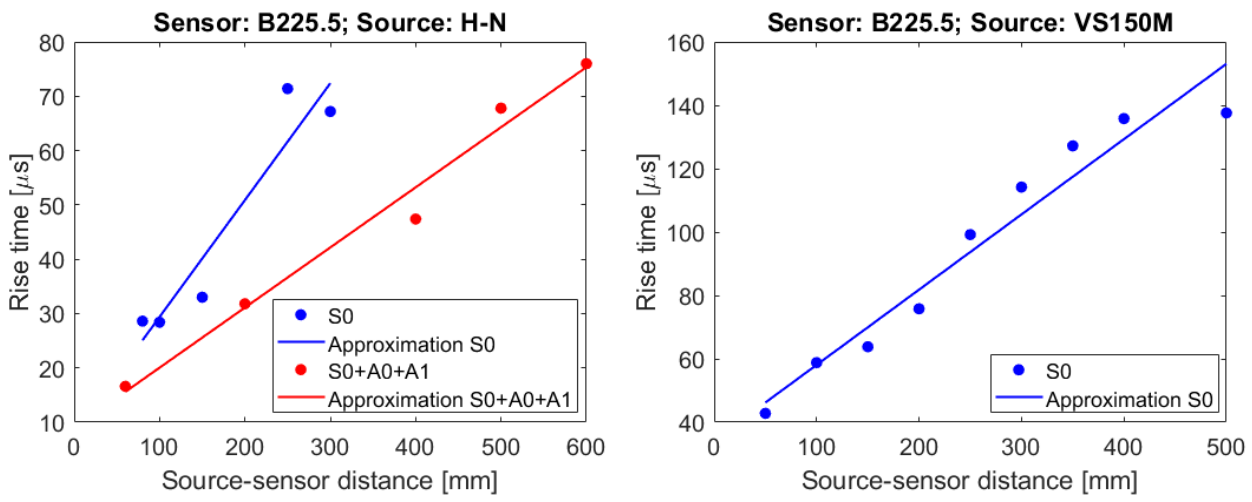

Fig. 12. Approximation of Rise time for sensor B225.5

are listed in Table 2. Time of arrival was assigned after employment of fictive threshold $35 \mathrm{~dB}$. It can be seen, that in the case of sensor B225.5 and mode of maximum S0, the value of slope is approximately the same regardless of source type used ( 0.22 for H-N source, 0.24 for VS150M source). Intercept of the line can serve to fit rise time of source itself, but some special attention should be paid by sources with greater rise times with regard to timefrequency content of the source.

Table 2. Experimental and theoretical stated slope of rise time vs. distance curve

\begin{tabular}{|c|c|c|c|c|}
\hline Sensor & Source & $\begin{array}{c}\text { Mode of } \\
\text { maximum }\end{array}$ & $\begin{array}{c}\text { Slope - experimental } \\
{[\boldsymbol{\mu \mathbf { s }} / \mathbf{m m}]}\end{array}$ & $\begin{array}{c}\text { Slope - theoretical } \\
{[\boldsymbol{\mu} \mathbf{s} / \mathbf{m m}]}\end{array}$ \\
\hline \multirow{3}{*}{ B225 } & \multirow{2}{*}{ H-N } & S0 & 0.22 & 0.25 \\
\cline { 2 - 5 } & VS150M & S0+A0+A1 & 0.1 & 0.1 \\
\hline VS30V & H-N & S0 & 0.24 & 0.25 \\
\hline
\end{tabular}




\section{Conclusion}

In presented paper the effect of wave propagation and characteristics of the sensors on detected acoustic emission signal was investigated. The significance of advanced data processing of captured AE signal for proper understanding of influences of fore-mentioned effects was demonstrated. By employment of CWT, group velocity dispersion curves and sensitivity curves of sensors used, wave modes and dominant frequency areas were successfully discriminated and direct wave was separated from reflected waves.

The mode corresponding to maximum amplitude differs with sensor type, but it can fluctuate also when 1 sensor used, if mode of maximum changes with position according to phase shifting. Attenuation curves from extracted amplitudes were constructed for a near field. It was revealed, that maximum amplitudes in a far field were extracted from superposition of reflections. Relevant attenuation curves in constructions with smaller lateral dimensions or in proximity of flanges, weld etc., if needed, can be constructed from a set of recorded waveform for each source-sensor position after separation of reflections according to presented methodology. Other AE parameters can be corrected with distance based on thoroughly analysed signal with respect to propagation effect and effect of a sensor. Correction of signal's rise time with distance was outlined.

This work was supported by STU - Program to support young researchers (2018/1382). Experimental part of research was realized in laboratory of Welding Research Institute - Industrial Institute of SR. Support is acknowledged.

Author would like to express sincere thanks to Mr. Ján Crha and Michal Šofer from VŠB-TU Ostrava for huge help by advising and securing experimental technique.

\section{References}

1. A. Vinogradov, A. Lazarev, M. Linderov, A. Weidner a H. Biermann, Kinetics of deformation processes in high-alloyed cast transformation-induced plasticity/twinninginduced plasticity steels determined by acoustic emission and scanning electron microscopy: Influence of austenite stability on deformation mechanisms. Acta Materialia 61, 2434-2449 (2013)

2. C. U. Grosse, Acoustic Emission Testing, (Berlin Heidelberg: Springer-Verlag, 2018)

3. K. M. Holford, M. J. Eaton, J. J. Hensman, R. Pullin, S. L. Evans, N. Dervilis a K. orden, A new methodology for automating acoustic emission detection of metallic fatigue fractures in highly demanding aerospace enviroments: An overview. Prog. in Aerosp. Scien. 90, 1-11, (2017)

4. J. L. Rose, Ultrasonic Guided Waves in Solid Media, Cambridge: Cambridge University Press, (2014)

5. T. N. Grigsby a E. J. Tajchman, Properties of Lamb Waves Relevant to the Ultrasonic Inspection of Thin Plates. IRE Tran. Utrason. Eng. 8, 26-33, (1961)

6. M. H. Sadd, Wave Motion and Vibration in Continuous Media. Kingston: University of Rhode Island, (2009)

7. M. Šofer, J. Crha a H. Zengerle, From Near Field To Far Field And Beyond. rev. 32nd Eu. Conf. on AET, (Prague, 2016)

8. J. Rayleigh, The Theory of Sound. (New York: Dover Publications, 1945) 
9. Nedeštruktívne skúšanie. Akustická emisia. Skúšanie tlakových zariadení počas preberacej skúšky. Planárne umiestnenie akustického emisného zdroja, STN EN 14584, (2013)

10. M. Šofer, P. Ferfecki a J. Neugebauer, Effect of the shape of geometric discontinuity on nature of Rayleigh wave back reflection. rev. EAN 2016 - 54th Conf. on Exp. Stress An., 242-248, (2016)

11. M. Šofer, P. Ferfecki a P. Šofer, Experimental construction of Lamb wave dispersion curves in plates. rev. EAN 2017 - 55th Conf. on Exp. Stress An., 242-248, (2017)

12. P. Šofer, M. Šofer a P. Ferfecki, Qualitative analysis of different Lamb wave excitation methods. rev. EAN 2017 - 55th Conf. on Exp. Stress An., 256-263, (2017)

13. M. A. Hamstad, A. O'Gallagher a J. Gary, A wavelet transform applied to acoustic emission signals: Part1: Source identification, J. AE 20, 39-61, (2002)

14. F. Dahmene, S. Yaacoubi, M. E. Mountassir, N. Bendaoud, C. Langlois a O. Bardoux, On the modal acoustic emission testing of composite structure. J. comp. struct. 140, 446-452 (2016).

15. J. Jiao, C. He, B. Wu, R. Fei a X. Wang, Application of wavelet transform on modal acoustic emission source location in thin plates with one sensor. In. Jour. of Press. Vess. and Pip. 81, 427-431 (2004).

16. M. Šofer, P. Ferfecki, M. Fusek, P. Šofer a D. Ličková, Numerical and Experimental Prediction of Lamb Wave Scattering from Horizontal Notch. rev. 56th Conf. on Exp. Stress. An. (2018)

17. S. Santhanam a R. Demirli, Reflection of Lamb waves obliquely incident on the free edge of a plate. Ultrasonics 53, 271-282 (2012)

18. I. G. Scott, Basic acoustic emission, London: Gordon and Breach Science Publishers, (1990) 